\title{
Hausarztmedizin: attraktive Zukunftsaussichten
}

\author{
Das schlechte Image der Hausarztmedizin ist überholt. Neue Entwicklungen haben \\ zu vielen Vorteilen gegenüber der Tätigkeit als Spezialarzt oder im Spital geführt. \\ Die Hausarztmedizin sollte in die Karriereplanung einbezogen werden.
}

Heini Zürcher

Korrespondenz:

Dr. med. Heini Zürcher

Argomed Ärzte AG

Bahnhofstrasse 24

CH-5600 Lenzburg

Tel. 0564415557

Fax 0564416255

heinrich.zuercher[at]hin.ch
Klagen über Überlastung, Notfalldienste und schlechte Entlohnung halten viele junge Ärzte ab, Hausarztmedizin als Weiterbildungsziel in Betracht zu ziehen. Die schlechten Arbeitsbedingungen gehören aber der Vergangenheit an. Die Hausarztmedizin erfüllt die heutigen Wünsche an die Lebensgestaltung optimal. Junge Ärzte sollten sie unbedingt als Berufsziel evaluieren.

\section{Genügende Auslastung ohne Überlastung}

Kein Zweifel: die Hausärzte werden mit Terminanfragen überhäuft. Von eigentlichen Notfällen abgesehen, dürfen wir solche Anfragen auch negativ beantworten. Für die Fehler der Politik in den letzen Jahrzehnten sind wir nicht verantwortlich und sollen sie nicht ausbaden, indem wir bis zur Erschöpfung arbeiten. Der Hausärztemangel hat den Vorteil, dass Hausärzte nicht fürchten müssen, zu wenig ausgelastet zu sein.

Die zentralen Notfallpraxen verbessern die Lebensbedingungen stark. Sie sind mehrheitlich den Notfallstationen von Spitälern angegliedert. Hier absolvieren die Hausärzte ihren Notfalldienst mit geregelten Arbeitszeiten und geregelter Entschädigung. Ausser in abgelegenen Gegenden ist der Notfalldienst rund um die Uhr bereits Vergangenheit.

\section{Gute Vereinbarkeit von Arbeit und Familie}

Ein Trend sind Gruppenpraxen. Hausärztinnen und Hausärzte schliessen sich zusammen. Administration und Betriebsführung können verteilt oder ganz abgegeben werden. IT-Systeme mit elektronischer Krankengeschichte sind selbstverständlich. Teilzeitarbeit ist fast zur Regel geworden. Neben der selbständigen Tätigkeit gibt es die Anstellung im Fixlohn oder mit Umsatzbeteiligung. Ein wesentlicher Vorteil gegenüber der Spitaltätigkeit ist die Selbstbestimmung über das $\mathrm{Ar}$ beitsumfeld, die Arbeitszeiten und die Ferien.

\section{Spannende Tätigkeit «mitten im Leben»}

Kaum ein anderer Bereich bringt Ärzte so nahe an das Leben anderer Menschen. Hausärzte beschäftigen sich mit dem Menschen und nicht nur mit einem Organsystem. Der langjährige Kontakt mit den Patienten, die Anerkennung durch sie hinterlässt viel Zufriedenheit.

\section{Verbessertes Einkommen}

Dank den Aktivitäten der Hausärzteorganisationen und dem Entgegenkommen des Bundesrats nähert sich das Einkommen dem der meisten Spezialisten, auch wenn es nicht ganz erreicht wird.

\section{Ausbildungscurriculum und Praxisassistenz}

Vorbei ist die Zeit, als angehende Hausärzte die Assistenzstellen mühsam suchen und häufig den Wohnort wechseln mussten. Vorbei auch die Zeit, als Jungärzte am ersten Tag in einer Praxis unvorbereitet unter die kalte Dusche gerieten. Weiterbildungs-Curricula und Praxisassistenzen sorgen für Abhilfe.

\section{Universitäre Hausarztmedizin}

Die Institute für Hausarztmedizin bringen schon Studierende mit der Hausarztmedizin in Kontakt. Ganz besonders kümmern sie sich um die lange vernachlässigte Forschung über die Grundversorgung. Sie beraten auch über den Einstieg in die Hausarztmedizin.

\section{Wir-Gefühl in der Hausarztbewegung}

Lokale, kantonale und gesamtschweizerische Organisationen fördern den gegenseitigen Austausch und die gegenseitige Unterstützung. Hier ist auch die HausarztInitiative entstanden, welche die medizinische Grundversorgung jetzt als Pflicht für die Behörden in der Bundesverfassung verankert hat. Wer Lust hat, kann mannigfache Tätigkeiten innerhalb der innovativen Organisationen übernehmen, zu denen auch Argomed gehört («bringt Ärzte weiter»). Den Engagierten winken ganz neue Erkenntnisse und neue Perspektiven. Die Organisation Junge Hausärzte Schweiz (JHaS) organisiert spannende Kongresse und lustvolle Treffen, bei denen das soziale Leben und das Vergnügen nicht zu kurz kommen.

\section{Hausärzte als Ausbildner}

In Einzeltutoriaten erleben Medizinstudenten die Hausarzttätigkeit. Studenten bewerten diese Tutoriate regelmässig als die weitaus besten Lehrveranstaltungen. Diese Tätigkeit ist für die Hausärzte sehr befriedigend und wird auch von den involvierten Patienten sehr geschätzt. Die Betreuung von Assistenzärzten im Rahmen der Praxisassistenz erlaubt den gegenseitigen Austausch und ist für beide Seiten spannend, lehrreich und äusserst befriedigend.

\section{Fazit}

Das verstaubte Image der Hausarztmedizin ist überholt. Die Hausarztmedizin bietet Berufseinsteigerinnen und -einsteigern attraktive Perspektiven und sollte unbedingt in die Planung der Weiterbildung einbezogen werden. 